
(c) (7) (8) Este trabalho está licenciado com uma Licença Creative Commons - Atribuição-NãoComercial 4.o Internacional. 
A REBEH - Revista Brasileira de Estudos da Homocultura é um periódico editado pela Associação Brasileira de Estudos da Homocultura (ABEH), que tem por objetivo a publicação de artigos, entrevistas, documentos, resenhas, trabalhos artísticos, ensaios, relatos de experiência e dossiês temáticos, que contemplem os estudos sobre gênero, sexualidade, raça, etnia e diversidades que interseccionem os marcadores sociais da diferença e posições ao avesso da norma.

Editoras Chefe (Gestão 2019-2020)

Prof $^{a}$ Dr $^{\mathrm{a}}$ Bruna Andrade Irineu (UFMT)

Prof. Dr. Moisés Alessandro Lopes (UFMT)

Prof. Dr. Danie Marcelo de Jesus (UFMT)

Editores Associados (Gestão 2019-2020)

Prof $^{\mathrm{a}} \mathrm{Dr}^{\mathrm{a}}$ Luma N. de Andrade (UNILAB)

Prof $^{a}$ Dra $^{a}$ Fran Demétrio (UFRB)

Prof. Dr. Marcos Aurélio da Silva (UFMT)

Prof $^{a}$ Dr $^{\mathrm{a}}$ Tatiana Lionço (UnB)

Prof. Dr. Márcio Neman (UFMT)

Prof. Dr. Marco José Duarte (UFJF)

Prof. Dr. Guilherme Passamani (UFMS)

Prof. MsC. Pablo Rocon (UFMT)

Prof. MsC. Gabriel Oliveira (IFMT)

Editor Júnior (Gestão 2019-2020)

Ayrton Senna S. do Amaral (UFMT)

\section{Imagem da Capa}

1 e 3 - II Marcha Nacional contra a Homofobia, Brasília;

2 - Parada do Orgulho LGBT, São Paulo;

Autoria: Bruna Andrade Irineu

Técnica: Fotografia digital

Diagramação

Ayrton Senna S. do Amaral e Bruna

\section{Revisão Textual}

Bruna Andrade Irineu, Ayrton Senna S. Amaral, Felipe Bruno M. Fernandes, Raíssa Lé, Barbara Alves e Dhan Tripoldi.

\section{Conselho Editorial}

Prof $^{a}$ Dr $^{\mathrm{a}}$. Ana Cristina Santos (UFAL)

Dd $^{a}$ Anahí Guedes (UFSC)

Prof $^{a}$ Dr $^{\text {a }}$ Berenice Bento (UnB)

Prof $^{\mathrm{a}}$ Dr $^{\mathrm{a}}$ Caterina Rea (UNILAB)

Prof. Dr. Carlos Eduardo de O. Bezerra

(UNILAB)

Prof $^{\mathrm{a}} \mathrm{Dr}^{\mathrm{a}}$ Cristina Vianna M. dos Santos

(UFT)

Prof ${ }^{\mathrm{a}} \mathrm{Dr}^{\mathrm{a}}$ Fátima Lima (UFRJ)

Prof. Dr. Felipe Bruno M. Fernandes (UFBA)

Prof. Dr. Guilherme Almeida (UFRJ)

Prof $^{\mathrm{a}} \mathrm{Dr}^{\mathrm{a}}$ Jaqueline de Jesus (IFRJ)

Prof. Dr. Horácio Costa (USP)

Prof $^{a}$ Dr $^{\mathrm{a}}$ Miriam Grossi (UFSC)

Prof. Dr. Sam Boucier (Université Lille III)

Prof $^{\mathrm{a}} \mathrm{Dr}^{\mathrm{a}}$ Suely Messeder (UNEB)

\section{Comitê de Avaliadores 2019}

Bruna Irineu; Vinicius Kabral; Ayrton Amaral; Murilo Peixoto; Adriana Salles; Rubenilson Araújo; Thiago Coacci; Rafael Noleto; Hugo Menezes Neto; Felipe Fernandes; Moisés Lopes; Marco José Duarte. 
REBEH: Revista Brasileira de Estudos da Homocultura/ Associação Brasileira de Estudos da Homocultura - ABEH, Universidade Federal da Integração da Lusofonia AfroBrasileira, UNILAB. vol. 2, n. 4 (2019)- . - Redenção/Ceará: UNILAB: ABEH, 2019v. il. : $26 \mathrm{~cm}$

Trimestral, 2019.

Texto em Português

Modo de Acesso:

$<$ http://www.revistas.unilab.edu.br/index.php/rebeh/index> ISSN 2595-3206 (Online)

1. Estudos de Gênero - Periódicos. 2. Diversidade - Periódicos.

I. Associação Brasileira de Estudos da Homocultura - ABEH. II. Universidade Federal da Integração da Lusofonia Afro-Brasileira, UNILAB. III. Título: REBEH: Revista Brasileira de Estudos da Homocultura. 